\section{Vorbeugen besser als Heilen}

Eine konsequente medizinische Behandlung kann Diabetespatienten vor Erblindung, Amputationen, Nierenversagen, Herzinfarkt und Schlaganfall bewahren. Der deutliche Rückgang dieser Folgeerkrankungen eines Diabetes konnte jetzt erstmals für die USA dokumentiert werden. Er wird jedoch von einem weiteren Anstieg der Diabetes-Erkrankungszahlen auch in Deutschland überschattet. Das teilte die Deutsche Gesellschaft für Endokrinologie (DGE) anlässlich einer im New England Journal of Medicine veröffentlichten Studie (Gregg EW et al.) mit.

Mitarbeiter der Centers for Disease Control and Prevention haben jüngst berichtet, dass in den Vereinigten Staaten die Zahl der Herzinfarkte bei Diabetespatienten in den letzten beiden Jahrzehnten um 2/3 (-67,8\%) zurückgegangen ist. Bei Schlaganfällen $(-52,7 \%)$ und Amputationen $(-51,4 \%)$ haben sich die Zahlen mehr als halbiert. Auch Todesfälle infolge eines diabetischen Komas (-64,4\%) sind deutlich seltener geworden. „Das sind eindrucksvolle Zahlen, die den heute hohen Stan- dard der medizinischen Diabetesbehandlung aufzeigen“, sagt Professor Dr. med. Dr. h. c. Helmut Schatz, DGE-Pressesprecher aus Bochum, der davon ausgeht, dass sich auch in Deutschland die Situation für Diabetespatienten verbessert hat.

„Anders als es in den USA der Fall war, sind hierzulande so gut wie alle Menschen krankenversichert und haben damit Anspruch auf eine kostenlose Versorgung“, sagt Schatz. „Wir haben heute nicht nur Medikamente, die Blutzucker, Cholesterin und Bluthochdruck effektiv senken“, so der Experte: „Zur Versorgung der Patienten gehören auch regelmäßige augenärztliche Untersuchungen, Überwachung des Herz-Kreislaufsystems, der Nieren und eine Fußpflege.“ Außerdem würden die Patienten immer auch zu einer Änderung des Lebensstils mit gesunder Ernährung, mehr Bewegung und Sport und gegebenenfalls einer Gewichtsreduktion motiviert. Nikotinverzicht gehört ebenfalls zu den wichtigen Allgemeinmaßnahmen.

Gleichwohl widersprechen die erhobenen relativen Zahlen den täglichen Erfahrun- gen von Klinikärzten und niedergelassenen Medizinern. Die Zahl der Menschen mit Diabetes ist in den letzten 2 Jahrzehnten in den USA und auch in Deutschland stark angestiegen. „Wir sehen immer mehr Menschen, die als Folge von Übergewicht und Bewegungsmangel an einem Typ-2-Diabetes erkranken“, stellt Schatz fest. Dies unterstreiche die enorme Wichtigkeit der Diabetesprävention. „Es gibt eine Reihe einfacher und effektiver Maßnahmen, die immer wieder am fehlenden Willen in der Politik scheitern“, kritisiert der Experte. Das wären beispielsweise eine Ampelkennzeichnung für Nahrungsmittel, die verbraucherfreundlicher sei als der jetzt obligatorische Nährwertkasten mit Zahlenangaben wie „pro 100 Gramm“, oder höhere Steuern für zuckerhaltige Getränke.

Ein weiterer effektiver, wenn auch aufwendiger Ansatz wären Präventionsmanager, die Hoch-Risiko-Personen regelmäßig persönlich beraten. „So wichtig die Erfolge bei der Diabetesbehandlung auch sind, die Therapie ist beim Typ-2-Diabetes kein Ersatz für die Prävention“, sagt Schatz. „Und vorbeugen ist bekanntlich besser als heilen.“

Pressemitteilung Deutsche Gesellschaft für Endokrinologie (DGE), 21.5.2014

durch rechtzeitig angewandte, gezielte präventive oder therapeutische Maßnahmen die Erkrankung und damit auch schwere Folgeschäden verhindern oder zumindest hinauszögern lassen“, sagt Matthias Schulze, Leiter der Abteilung Molekulare Epidemiologie am DIfE. Zu den Folgeerkrankungen zählen u.a. Schädigungen der Augen und Nieren, ein Herzinfarkt oder Schlaganfall. jähriger, dessen Vater oder Mutter an Typ-2-Diabetes erkrankt ist, ein Risiko wie ein Sechzigjähriger ohne Vorbelastung“, erklärt Hans-Georg Joost, wissenschaftlicher Direktor des DIfE. Bei Vorliegen einer familiären Belastung sei also eine besonders intensive Prävention durch eine Ernährungs- und Lebensstilumstellung angezeigt, sagt der Mediziner und Pharmakologe weiter. „Nutzer des Tests können dabei anhand der vergebenen Punktzahl abschätzen, inwieweit sie ihr persönliches Risiko selbst günstig beeinflussen können, indem sie zum Beispiel ihren Taillenumfang reduzieren oder ihren Vollkornbrotverzehr erhöhen“, ergänzt Kristin Mühlenbruch, Erstautorin der oben zitierten Studie.

„Das Gute an Typ-2-Diabetes ist, dass sich

\section{Einfache Anwendung bei}

Vorsorgeuntersuchung möglich

Die Wissenschaftler des DIfE würden es daher begrüßen, wenn Allgemeinmediziner den Patientenfragebogen zusammen mit einer einfachen Blutzuckeruntersuchung im Rahmen einer Vorsorgeuntersuchung nutzten, um das Risikoprofil einer Person ab dem 35. Lebensjahr zu bestimmen. Präventionsmaßnahmen ließen sich so sinnvoll auf die Gruppe der Hochrisikopersonen begrenzen. Darüber hinaus sei der Test für eine anschließende, quantitative Erfolgskontrolle nutzbar.

Pressemitteilung Deutsches Institut für Ernährungsforschung Potsdam-Rehbrücke (DIfE), 19.5.2014 


\section{Zu viel Proteine sind schlecht fürs Kind}

Die Ernährung während der Schwangerschaft und in der frühen Kindheit hat Folgen für das ganze Leben. Sie wirkt sich auf das kindliche Wachstum aus und beeinflusst lebenslang den Stoffwechsel. „Einer der besten Indikatoren, um das spätere Risiko vorherzusagen, übergewichtig zu werden, ist die Gewichtszunahme während des ersten Lebensjahres“, sagt Prof. Berthold Koletzko, Leiter der Abteilung Stoffwechsel und Ernährung am Dr. von Haunerschen Kinderspital der LMU.

Wie entscheidend dabei der Proteingehalt der Nahrung im Säuglingsalter ist, konnte Koletzko nun mit seinem Team in einer Studie nachweisen, deren Ergebnisse in der Zeitschrift American Journal of Clinical Nutrition veröffentlicht wurden. Die Wissenschaftler verfolgten den BodyMass-Index (BMI) und die Gewichtszunahme von 2 Gruppen von Kindern, die als Babys Säuglingsnahrung mit unterschiedlichem Proteingehalt erhielten. „Kinder, deren Säuglingsnahrung einen höheren Proteingehalt hatte, hatten im Alter von 6 Jahren einen deutlich höheren BMI. Ihr Risiko, übergewichtig zu werden, war fast zweieinhalb mal höher als das jener Kinder, die proteinärmere Nahrung erhielten“, sagt Koletzko.

Langfristige Folgen für den Stoffwechsel Koletzko hat die Kinder erstmals im Jahr 2002 untersucht. In früheren Studien konnte Koletzko bereits zeigen, welche Folgen Säuglingsnahrung mit einem höheren Proteingehalt für die ersten beiden Lebensjahre hat: Die Kinder legten im ersten Jahr mehr an Gewicht zu und waren im Alter von 2 Jahren schwerer als Kinder, die Nahrung mit weniger Protein erhielten.

Die Folgeuntersuchung mit den Kindern, die inzwischen 6 Jahre alt waren, zeigte nun, wie langfristig die Ernährung im Säuglingsalter das Gewicht der Kinder bestimmt. „Das erhöhte Risiko, im Alter von 6 Jahren übergewichtig zu sein, deutet darauf hin, dass die höhere Proteinzufuhr bei Babys nicht nur zu einer schnelleren Gewichtszunahme führt. Offenbar sind damit auch langfristige Auswirkungen auf den Stoffwechsel verbunden“, sagt Koletzko.

Die Entwicklung der Säuglinge beider Gruppen wurde mit der Entwicklung ge-

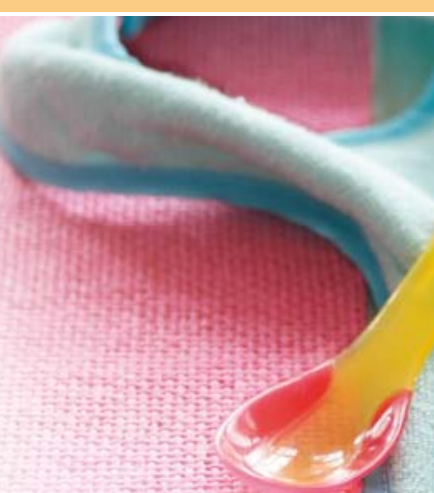

stillter Babys verglichen. Kinder, deren Nahrung dem Proteingehalt der Muttermilch entsprach, waren später auch in der ân Gewichtszunahme und bei der Entwick丞 lung des BMI mit gestillten Kindern ver氕 gleichbar.

„Eine optimale Ernährung von Säuglingen ist enorm wichtig, da sie die Grundlagen für die künftige Gesundheit legt“, sagt Koletzko. Die Studienergebnisse sind für die öffentliche Gesundheitsvorsorge von Bedeutung. „Momentan führen Säuglingsnahrung und ergänzende Nahrung für Babys zu Proteineinnahmen, die höher sind als empfohlen“, sagt Koletzko. „Die Empfehlung an Mütter, zu stillen und eine Reduktion des Proteingehalts in der Säuglingsnahrung sind wichtige Präventionsmaßnahmen, damit Kinder nicht übergewichtig werden.“

Pressemitteilung Ludwig-Maximilians-Universität München vom 18.3.2014

\section{Gutes Selbstmanagement senkt Mortalität}

Die Wissenschaftler vom Institut für Gesundheitsökonomie und Management im Gesundheitswesen (IGM) und vom Institut für Epidemiologie II (EPI II) am Helmholtz Zentrum München (HMGU) untersuchten gemeinsam mit Kollegen des Deutschen Diabetes-Zentrums (DDZ) in Düsseldorf den Zusammenhang des Selbstmanagements mit der Mortalität bei Patienten mit Typ-2-Diabetes. Die Ergebnisse wurden in Diabetes Care veröffentlicht. Das HMGU sowie das DDZ sind Partner im Deutschen Zentrum für Diabetesforschung (DZD).

Hoher Selbstmanagement-Index niedrige Mortalität

340 Studienteilnehmer mit Typ-2-Diabetes wurden bezüglich ihres Patientenverhaltens, welches beispielsweise regelmäßiges Kontrollieren des Blutzuckerspiegels, Führen eines Ernährungsplans oder körperliche Aktivität umfasst, befragt und daraus ein Selbstmanagement-Index ermittelt. Diesen Index setzte das Team um Prof. Rolf Holle und Michael Laxy in Zusammenhang mit der Sterblichkeit der Personen, die über einen Zeitraum von 12 Jahren erfasst wurde. Die Analyse ergab, dass Patienten mit einem guten Diabetes-Selbstmanagement, d.h. mit einem hohen Selbstmanagement-Index, ein deutlich geringeres Mortalitätsrisiko aufweisen, als Patienten mit einem niedrigen Selbstmanagement-Index. Dieser Zusammenhang besteht unabhängig von anderen Faktoren, die die Mortalität beeinflussen können, wie etwa Alter, Geschlecht, Begleiterkrankungen oder medikamentöse Therapien.

\section{Aktive Therapiebeteiligung}

der Patienten wichtig

„Die Ergebnisse zeigen, dass neben einer leitliniengerechten ärztlichen Behand- lung auch das Patientenverhalten eine große Bedeutung für den Krankheitsverlauf sowie den Behandlungserfolg hat", erklärt Holle, Leiter der Arbeitsgruppe Ökonomische Evaluation am IGM. „Patientenzentrierte Angebote, wie Diabetesschulungen und Informationsangebote, leisten daher einen wertvollen Beitrag für eine gute Patientenversorgung und sollten künftig weiter ausgebaut werden“.

Grundlage der analysierten Daten ist die KORA-A-Studie, die sich aus Teilnehmern von 2 früheren bevölkerungsbasierten Gesundheitsstudien und aus Patienten des KORA-Herzinfarktregisters aus dem Raum Augsburg zusammensetzt.

Pressemitteilung Helmholtz Zentrum München Deutsches Forschungszentrum für Gesundheit und Umwelt vom 26.3.2014 